\title{
ISOPERIMETRIC INEQUALITIES AND DIRICHLET FUNCTIONS OF RIEMANN SURFACES
}

\author{
JosÉ M. RodRÍGUEZ
}

\begin{abstract}
We prove that if a Riemann surface has a linear isoperimetric inequality and verifies an extra condition of regularity, then there exists a non-constant harmonic function with finite Dirichlet integral in the surface.

We prove too, by an example, that the implication is not true without the condition of regularity.
\end{abstract}

\section{Introduction.}

In this paper we study the relationship between linear isoperimetric inequalities and the existence of harmonic functions with finite Dirichlet integral on Riemann surfaces.

By $\mathcal{S}$ we denote a Riemann surface (whose universal covering space is the unit disk $\Delta$ ) endowed with its Poincaré metric, i.e. the metric obtained by projecting the Poincaré metric of the unit disk: $d s=2(1-$ $\left.|z|^{2}\right)^{-1}|d z|$. With this metric, $\mathcal{S}$ is a complete Riemannian manifold with constant curvature -1 . The only Riemann surfaces which are left out are the sphere, the plane, the punctured plane and the tori.

We shall say that a Riemann surface $\mathcal{S}$ satisfies a "linear isoperimetric inequality" (LII) if there exists a finite constant $h(\mathcal{S})$ so that for every relatively compact open set $G$ with smooth boundary we have

$$
A(G) \leq h(\mathcal{S}) L(\partial G)
$$

Here and from now on, $A, L, d$ and $B$ refer to Poincaré area, length, distance and open ball of $\mathcal{S}$.

There are connections between LII and some conformal invariants on Riemann surfaces: the bottom of the spectrum of the Laplace-Beltrami operator, $b(\mathcal{S})$, and the exponent of convergence $\delta(\mathcal{S})$ : 
Theorem A. ([Ch], [B, p. 228], [FR]). A Riemann surface $\mathcal{S}$ satisfies a linear isoperimetric inequality if and only if $b(\mathcal{S})>0$. In fact,

$$
\frac{1}{4} \leq b(\mathcal{S}) h(\mathcal{S})^{2} \quad \text { and } \quad b(\mathcal{S}) h(\mathcal{S})<\frac{3}{2} .
$$

The next result is a well known theorem of Elstrodt-Patterson-Sullivan:

Theorem B. [S, p. 333]. A Riemann surface $\mathcal{S}$ satisfies a linear isoperimetric inequality if and only if $\delta(\mathcal{S})<1$. In fact,

$$
b(\mathcal{S})= \begin{cases}\frac{1}{4}, & \text { if } 0 \leq \delta(\mathcal{S}) \leq \frac{1}{2}, \\ \delta(\mathcal{S})(1-\delta(\mathcal{S})), & \text { if } \frac{1}{2} \leq \delta(\mathcal{S}) \leq 1 .\end{cases}
$$

A theorem of Myrberg [ $\mathbf{T}$, p. 522] states that if $\delta(\mathcal{S})<1$ (if $\mathcal{S}$ satisfies a LII) then $\mathcal{S}$ has a Green's function $\left(\mathcal{S} \notin O_{G}\right.$ in the language of classification theory). If $\mathcal{S}$ is a plane domain (in fact, if $\mathcal{S}$ is a surface of almost finite genus [SN, p. 193]), $\mathcal{S}$ has Green's function if and only if $\mathcal{S}$ has a non-constant harmonic function with finite Dirichlet integral [SN, p. 194] ( $\mathcal{S} \notin O_{H D}$ in the language of classification theory).

One would like to understand the relationship between the classes $O_{H D}$ and $\mathcal{B}$ (the Riemann surfaces which do not satisfy a LII). As we have said above, in the case of surfaces of almost finite genus, $O_{G}=$ $O_{H D} \subset \mathcal{B}$. The inclusion is strict, as it is shown by the example $\mathcal{S}_{0}=$ $\Delta \backslash\left(\cup_{k=1}^{\infty}\left\{2^{-k}\right\} \cup\{0\}\right): \mathcal{S}_{0} \notin O_{G}$ because it is a plane domain whose boundary has positive logarithmic capacity [T, p. 81]; $\mathcal{S}_{0} \in \mathcal{B}$ because $\cup_{k=1}^{\infty}\left\{2^{-k}\right\} \cup\{0\}$ is a discrete set with an accumulation point in $\Delta[\mathbf{F R}$, Theorem 4].

The inclusion $O_{H D} \subset \mathcal{B}$ is true, in general, with an extra hypothesis:

Theorem 1. Let $\mathcal{S}$ be a Riemann surface which satisfies a linear isoperimetric inequality. If there exists in $\mathcal{S}$ a set of disjoint simple closed curves $\left\{\gamma_{j}\right\}_{j=1}^{m}$, such that $\mathcal{S} \backslash \cup_{j} \gamma_{j}$ contains $n$ connected components of infinite area $\mathcal{S}_{1}, \ldots, \mathcal{S}_{n}$, then

$$
\operatorname{dim} H D(\mathcal{S}) \geq n .
$$

This inequality is the best possible.

Here $H D(\mathcal{S})$ denotes the (real) linear space of harmonic functions in $\mathcal{S}$ with finite Dirichlet integral.

The inclusion $O_{H D} \subset \mathcal{B}$ is not true in the general case, even with the extra customary hypothesis of bounded geometry $[\mathbf{K}]$, which in our 
context means that the injectivity radius $\iota(\mathcal{S})$ is positive. $\iota(\mathcal{S})$ is defined as

$$
\iota(\mathcal{S})=\inf \{\iota(p): p \in \mathcal{S}\},
$$

where $\iota(p)$ is the injectivity radius of the geodesic exponential map centered at $p$.

Theorem 2. There exists a Riemann surface $\mathcal{R} \in O_{H D}$, with $\iota(\mathcal{R})>$ 0 , which satisfies a linear isoperimetric inequality.

Acknowledgements. I would like to thank J. L. Fernández for many useful conversations about these results, and J. Llorente for his careful reading of the manuscript and for some helpful suggestions.

\section{Proof of Theorem 1.}

Without loss of generality, we can assume that $\left\{\gamma_{j}\right\}_{j=1}^{m}$ are simple closed geodesics. If this is not the case, we can substitute each curve by the geodesic in its same free homotopy class.

Let $\mathcal{S}_{k}$ be a component of infinite area of $\mathcal{S} \backslash \cup_{j} \gamma_{j}$, and let $\mathcal{S}_{k}^{*}$ be the Schottky double of $\mathcal{S}_{k}$ (see [AS, p. 26] for the definition).

Claim. $\mathcal{S}_{k}^{*}$ satisfies a linear isoperimetric inequality.

If the claim is true, the theorem of Myrberg [ $\mathbf{T}$, p. 522] states that $\mathcal{S}_{k}^{*}$ has Green's function. This implies that the Royden's harmonic boundary of $\mathcal{S}_{k}^{*}$ is not empty [SN, p. 166].

$\mathcal{S}_{k}^{*}$ is symmetrical with respect to $\partial \mathcal{S}_{k}$, a compact set which separates $\mathcal{S}_{k}^{*}$ in two connected components. Then the Royden's harmonic boundary of $S_{k}^{*}$ is also symmetrical with respect to $\partial \mathcal{S}_{k}$, and contains at least two points, one of them corresponding to $\mathcal{S}_{k}$ (one of them is in the closure of $\mathcal{S}_{k}$ in the Royden's compactification of $\mathcal{S}_{k}^{*}$ ).

This is true for $k=1, \ldots, n$. Therefore, the Royden's harmonic boundary of $\mathcal{S}$ contains at least $n$ points [SN, p. 191]. This is equivalent [SN, p. 166] to

$$
\operatorname{dim} H D(\mathcal{S}) \geq n \text {. }
$$

This inequality is the best possible:

Let $\mathcal{R}$ be the Riemann surface given by Theorem 2 ( $\mathcal{R}$ will be constructed without any mention to Theorem 1) and consider $\mathcal{R}_{n}$, a $n$ covering of $\mathcal{R}$ based in a closed simple geodesic $\gamma \subset \mathcal{R}$. $\mathcal{R}_{n}$ satisfies the hypothesis of this theorem and also $\operatorname{dim} H D\left(\mathcal{R}_{n}\right)=n$ (the Royden's harmonic boundary of $\mathcal{R}_{n}$ consists of $n$ points, because the Royden's harmonic boundary of $\mathcal{R}$ consists of one point $\left(\mathcal{R} \in O_{H D} \backslash O_{G}\right)$ [SN, p. 166]). 
To finish the proof of Theorem 1, we only need to prove the Claim:

By a geodesic domain in a Riemann surface we mean a connected domain $G$ with finite area, such that $\partial G$ consists of finitely many closed simple geodesics. $G$ does not have to be relatively compact since it may "surround" finitely many punctures.

The following lemma will be very useful:

Lemma. [FR, p. 168]. A Riemann surface satisfies a LII if and only if it satisfies LII for geodesic domains. Moreover, if $h$ and $h_{g}$ are, respectively, the usual and geodesic isoperimetric constants, then

$$
h_{g} \leq h \leq 2+h_{g} .
$$

Therefore, we must verify LII only for geodesic domains of $\mathcal{S}_{k}^{*}$. By the symmetry of $\mathcal{S}_{k}^{*}$ and the LII of $\mathcal{S}$, we just need to check this for geodesic domains which are symmetrical with respect to $\partial S_{k}$. Then, we must verify

$$
A(G) \leq c L\left(\partial_{0} G\right)
$$

for geodesic domains $G$ of $\mathcal{S}_{k}$, such that $\partial G \cap \partial \mathcal{S}_{k} \neq \varnothing$, where $\partial_{0} G$ means

$$
\partial_{0} G \equiv \partial G \backslash \partial \mathcal{S}_{k} .
$$

Consider the open sets $C_{t}=\left\{p \in \mathcal{S}_{k}: d\left(p, \partial \mathcal{S}_{k}\right)<t\right\}$ for positive $t$. Let $G_{t}$ be the geodesic domain "corresponding" to $C_{t}$ (each puncture or boundary curve of $G_{t}$ is freely homotopic to a boundary curve of $C_{t}$ ). If $G_{t}$ is empty for all positive $t$, then $\mathcal{S}_{k}$ is a doubly connected domain (a funnel), $\mathcal{S}_{k}^{*}$ is an annulus, and the claim is true with constant 1 . Then, we can assume that $G_{t}$ is connected and not empty for $t \geq t_{0}$. $G_{t}$ is non decreasing in $t$, and if $t_{1}<t_{2}$ are such that $A\left(G_{t_{1}}\right)<A\left(G_{t_{2}}\right)$, the constant curvature -1 and the Gauss-Bonnet theorem give $A\left(G_{t_{1}}\right)+2 \pi \leq$ $A\left(G_{t_{2}}\right)$.

This implies that there exists a positive number $T$ such that $G_{t}=G_{T}$ for all $t \geq T$, or $A\left(G_{t}\right) \rightarrow \infty$ as $t \rightarrow \infty$.

The first possibility is easy: there are only a finite number of geodesic domains. Without loss of generality, we can asssume that $A\left(G_{t}\right) \rightarrow \infty$ as $t \rightarrow \infty$.

Case 1. $A(G) \geq 2 h(\mathcal{S}) \ell$, with $\ell=\sum_{j=1}^{m} L\left(\gamma_{j}\right)$. In this case,

$$
2 h(\mathcal{S}) \ell \leq A(G) \leq h(\mathcal{S}) L(\partial G) \leq h(\mathcal{S})\left(L\left(\partial_{0} G\right)+\ell\right)
$$

and

$$
\ell \leq L\left(\partial_{0} G\right)
$$


Therefore,

$$
A(G) \leq 2 h(\mathcal{S}) L\left(\partial_{0} G\right) .
$$

Case 2. $A(G)<2 h(\mathcal{S}) \ell$.

Let $\Omega$ be a geodesic domain in $\mathcal{S}_{k}$ such that

$$
\partial \mathcal{S}_{k} \subset \partial \Omega \quad \text { and } \quad A(\Omega) \geq 2 h(\mathcal{S}) \ell .
$$

We can choose $\Omega$, for example, as the first geodesic domain $G_{t}$ satisfying $A\left(G_{t}\right) \geq 2 h(\mathcal{S}) \ell$.

We define

$$
\begin{gathered}
a \equiv \min \{L(\gamma): \gamma \text { closed simple geodesic, } \gamma \subset \bar{\Omega}\} \\
b \equiv \max \left\{L(\gamma): \gamma \text { closed simple geodesic, } \gamma \subset \partial_{0} \Omega\right\}
\end{gathered}
$$

Since $A(\Omega)>A(G)$ and $\Omega \cap G \neq \varnothing$, one of the two next possibilities holds:

Case 2.1. There exists a closed simple geodesic $\gamma \subset \bar{\Omega} \cap \partial_{0} G$. Then

$$
L\left(\partial_{0} G\right) \geq L(\gamma) \geq a .
$$

Case 2.2. There exists a closed simple geodesic $\eta$ in $\partial_{0} G$, which meets some $\gamma \subset \partial_{0} \Omega$.

Then, the Collar Lemma $[\mathbf{R}]$ says that $L(\eta) \geq 4 d_{0}$, where $d_{0}$ (the width of the greater collar of $\gamma$ ) satisfies

$$
\cosh d_{0} \geq \operatorname{coth} \frac{L(\gamma)}{2} \geq \operatorname{coth} \frac{b}{2},
$$

and

$$
d_{0} \geq D \equiv \operatorname{arccosh}\left(\operatorname{coth} \frac{b}{2}\right) .
$$

Randol $[\mathbf{R}]$ states the Collar Lemma if the surface is compact, but the same proof, without any change, works for a general Riemann surface.

Therefore,

$$
L\left(\partial_{0} G\right) \geq L(\eta) \geq 4 D .
$$

In both cases (2.1 and 2.2) $L\left(\partial_{0} G\right) \geq \min \{a, 4 D\} \equiv c_{0}$. Then

$$
A(G) \leq h(\mathcal{S})\left(L\left(\partial_{0} G\right)+\ell\right) \leq h(\mathcal{S})\left(L\left(\partial_{0} G\right)+\ell \frac{L\left(\partial_{0} G\right)}{c_{0}}\right)
$$


and

$$
A(G) \leq h(\mathcal{S})\left(1+\frac{\ell}{c_{0}}\right) L\left(\partial_{0} G\right) .
$$

Obviously, $\ell \geq a \geq c_{0}$ and $1+\ell / c_{0} \geq 2$. Therefore, in any case,

$$
A(G) \leq h(\mathcal{S})\left(1+\frac{\ell}{c_{0}}\right) L\left(\partial_{0} G\right)
$$

Consequently,

$$
h\left(\mathcal{S}_{k}^{*}\right) \leq 2+h_{g}\left(\mathcal{S}_{k}^{*}\right) \leq 2+h(\mathcal{S})\left(1+\frac{\ell}{c_{0}}\right),
$$

and the proof of Theorem 1 is now complete.

\section{Proof of Theorem 2.}

The desired Riemann surface $\mathcal{R}$ will be obtained with the help of a graph $G$. We will construct this graph in three steps.

In the set of vertices of any connected graph we can define a natural distance:

$$
d(p, q)=\inf \{\text { length of the paths from } p \text { to } q\} .
$$

This will be "the distance" in all graphs of this section.

First, let $T$ be the infinite complete binary tree with root $r_{0}$. Secondly, let $V_{n}$ be the subset of $2^{n}$ vertices of $T$ at distance $n$ of $r_{0}$. We can construct new graphs $G_{n}(n \geq 1)$ with vertices $V_{n}$. In $G_{1}$ there is one edge between the two vertices of $V_{1}$. The edges of $G_{n}(n \geq 2)$ are chosen as follows: $2^{n}-1$ vertices of $V_{n}$ are connected by a complete binary tree with $2^{n-1}$ leaves and with root $r_{n}$ (in any way); we add another edge between $r_{n}$ and the last vertex $v_{n}$ of $V_{n}$. In this way, the degree of the vertices of $G_{n}$ is one (if the vertex is a leave) or three (if the vertex is not a leave). The leaves are at distance $n-1$ of $r_{n}$, except for $v_{n}$ which is at distance 1. Hence, the diameter of $G_{n}$ is $2 n-2$, if $n \geq 2$.

Finally, we are ready to construct the graph $G$. The vertices of $G$ are the vertices of $T$. The edges of $G$ are the union of the edges of $T$ and the edges of $G_{n}$, for all $n \geq 1$. The root $r_{0}$ of $G$ has degree two. The other vertices of $G$ have degree four or six.

To build up our Riemann surface $\mathcal{R}$, modelled upon the graph $G$, we will need the so called Löbell $Y$-pieces, which are a standard tool for constructing Riemann surfaces. A clear description of these $Y$-pieces and their use is given in [C, Chapter X.3]. 
A Löbell $Y$-piece is a three-holed sphere, endowed with a metric of constant negative curvature -1 , so that the boundary curves are geodesics. We also require that the lengths of the boundary curves are the same, say $2 \alpha$, and the distance between any two of these boundary curves is $\beta$, say. Then $\alpha$ and $\beta$ are related by

$$
\sinh \left(\frac{\alpha}{2}\right) \sinh \left(\frac{\beta}{2}\right)=\frac{1}{2} .
$$

This is the unique restriction on $\alpha$ and $\beta$. See [C, p. 248] for details.

Fix $\alpha$ and $\beta$ satisfying the above relation.

A $X$-piece (*-piece) is a four-holed (six-holed) sphere, endowed with a metric of curvature -1 , so that the boundary curves are geodesics of the same length $2 \alpha$. We can construct these pieces, for example, joining two (four) $Y$-pieces, by identifying corresponding boundary curves.

If we now put together these pieces following the combinatorial design of $G$, with the $X$-pieces ( ${ }^{*}$-pieces) in the place of the vertices of degree four (six), we obtain a complete surface of constant negative curvature -1 .

The only non-standard vertex is $r_{0}$, which has degree two. There is not problem if we forget $r_{0}$ and consider that the two vertices of $V_{1}$, are connected by a double edge.

Since we have used only two distinct pieces to build up $\mathcal{R}$, it is trivial to see that $\iota(\mathcal{R})>0$.

First of all, we will prove that $\mathcal{R} \in O_{H D}$. Let $u$ be a harmonic function in $\mathcal{R}$ with finite Dirichlet integral. Without loss of generality we can assume that $u$ is a bounded function [AS, p. 203] [SN, p. 178]. We want to verify that $u$ is constant.

If $u$ has limit at infinity, there is a point $p$ in $\mathcal{R}$ such that $u(p)$ is the maximum or the minimum of $u$ in $\mathcal{R}$. The maximum principle implies that $u$ is constant.

If $u$ is non-constant and has not limit at infinity, we can assume that $u$ is positive and

$$
\limsup _{z \rightarrow \infty} u(z)>4 \quad \text { and } \quad \liminf _{z \rightarrow \infty} u(z)<1 .
$$

The maximum (minimum) principle implies that each connected component of the set $\{u>4\}(\{u<1\})$ is not a relatively compact set of $\mathcal{R}$.

This implies that, for each $n \geq n_{0}$, there exist points $p_{n}, q_{n}$ in the pieces of $\mathcal{R}$ corresponding to $G_{n}$, such that

$$
u\left(p_{n}\right)>4 \quad \text { and } \quad u\left(q_{n}\right)<1 .
$$


Since $u$ is a positive harmonic function, the Harnack's Theorem says that there exists a positive number $\varepsilon<\iota(\mathcal{R})$, independent of $n$, such that,

$$
\begin{aligned}
& u(z) \geq 3, \quad \text { for all } \quad z \in B\left(p_{n}, \varepsilon\right) \\
& u(z) \leq 2, \quad \text { for all } \quad z \in B\left(q_{n}, \varepsilon\right) .
\end{aligned}
$$

Let the manifold with boundary $\mathcal{R}_{n}$ be the union of the pieces in $\mathcal{R}$ corresponding to the vertices $V_{n}$ of $G_{n}$. We need a geodesic $\gamma_{n}$ between $p_{n}$ and $q_{n}$, completely contained in $\mathcal{R}_{n}$, which minimizes distance inside $\mathcal{R}_{n}$. To prove the existence of such geodesic, consider the Riemann surface $\Omega$

$$
\Omega \equiv\left\{z \in \mathbb{C}: 1<|z|<v^{2}\right\},
$$

where the constant $v$ is chosen so that the geodesic $\{|z|=v\}$ has length $2 \alpha$, the length of each boundary curve of $\mathcal{R}_{n}$. If we join a copy of $\Omega_{0} \equiv$ $\{1<|z| \leq v\}$ in each boundary curve of $\mathcal{R}_{n}$, we obtain a new Riemann surface $\mathcal{R}_{n}^{0}$. Since $\mathcal{R}_{n}^{0}$ is complete, there is a geodesic $\gamma_{n}$ between $p_{n}$ and $q_{n}$ such that the length $L_{n}$ of $\gamma_{n}$ is equal to the distance between $p_{n}$ and $q_{n} . \gamma_{n}$ is "completely contained in $\mathcal{R}_{n}$ ", because if $\gamma$ enters in some copy of $\Omega_{0}$, it lies there forever.

Consider now the Fermi coordinates $(r, t)\left[\mathbf{C}\right.$, p. 247], where $r \in\left[0, L_{n}\right]$ describes the curve $\gamma_{n}$, and $t \in[-\varepsilon, \varepsilon]$ describes the orthogonal geodesics to $\gamma_{n}$.

Observe that if we choose

$$
\varepsilon<\frac{1}{2} \operatorname{arc} \cosh \sqrt{\cosh \iota(\mathcal{R})}<\iota(\mathcal{R}),
$$

$\left(0, L_{n}\right) \times(-\varepsilon, \varepsilon)$ corresponds injectively to a region $\Lambda_{n} \subset \mathcal{R}$. Let us denote by $\pi$ this correspondence.

Assume that there exists two points $\left(r_{1}, t_{1}\right) \neq\left(r_{2}, t_{2}\right)$ in $\left(0, L_{n}\right) \times$ $(-\varepsilon, \varepsilon)$, corresponding to the same $p \in \Lambda_{n}$. By the definition of $\iota(\mathcal{R})$, it is not possible that $\left(r_{2}, t_{2}\right) \in B\left(\left(r_{1}, 0\right), \iota(\mathcal{R})\right)$. This implies $\left|r_{1}-r_{2}\right|>2 \varepsilon$, because if $d \equiv d\left(\left(r_{2}, t_{2}\right),\left(r_{1}, 0\right)\right)$, hyperbolic trigonometry [F, p. 92] gives

$$
\cosh \left(r_{2}-r_{1}\right) \cosh t_{2}=\cosh d \geq \cosh \iota(\mathcal{R})>\cosh ^{2}(2 \varepsilon),
$$

and we have

$$
\cosh \left(r_{2}-r_{1}\right) \cosh \varepsilon>\cosh \left(r_{2}-r_{1}\right) \cosh t_{2}>\cosh (2 \varepsilon) \cosh \varepsilon .
$$

Then

$$
\left|r_{2}-r_{1}\right|>2 \varepsilon,
$$


and

$$
\begin{aligned}
d\left(\pi\left(r_{1}, 0\right), \pi\left(r_{2}, 0\right)\right) & \leq d\left(\pi\left(r_{1}, 0\right), \pi\left(r_{1}, t_{1}\right)\right)+d\left(\pi\left(r_{2}, t_{2}\right), \pi\left(r_{2}, 0\right)\right) \\
& =t_{1}+t_{2}<2 \varepsilon .
\end{aligned}
$$

But $\left|r_{1}-r_{2}\right|>2 \varepsilon$ and $d\left(\pi\left(r_{1}, 0\right), \pi\left(r_{2}, 0\right)\right)<2 \varepsilon$ contradict that $\gamma_{n}$ minimizes length between $p_{n}$ and $q_{n}$.

Therefore, $\left(0, L_{n}\right) \times(-\varepsilon, \varepsilon)$ corresponds injectively to a region $\Lambda_{n} \subset \mathcal{R}$.

It is easy to see that for all $t \in(-\varepsilon, \varepsilon)$, if $\gamma_{n}^{t} \equiv\left\{\pi(r, t): 0 \leq r \leq L_{n}\right\}$,

$$
\begin{aligned}
1 & \leq u(\pi(0, t))-u\left(\pi\left(L_{n}, t\right)\right)=\left|\int_{\gamma_{n}^{t}} \nabla u d s\right| \\
& \leq \int_{\gamma_{n}^{t}}|\nabla u| d s \leq\left(\int_{\gamma_{n}^{t}}|\nabla u|^{2} d s\right)^{1 / 2}\left(L\left(\gamma_{n}^{t}\right)\right)^{1 / 2} .
\end{aligned}
$$

But the metric in Fermi coordinates is expressed by

$$
d s^{2}=\cosh ^{2} t d r^{2}+d t^{2},
$$

and so

$$
L\left(\gamma_{n}^{t}\right)=\int_{0}^{L_{n}} \cosh t d r=L_{n} \cosh t<L_{n} \cosh \varepsilon \leq 2 D n \cosh \varepsilon,
$$

if $D$ is the maximum of the diameters of the $X$-pieces and the *-pieces, because the diameter of $G_{n}$ is $2 n-2$. This gives

$$
\int_{\gamma_{n}^{t}}|\nabla u|^{2} d s \geq \frac{1}{2 D n \cosh \varepsilon}
$$

and

$$
\int_{\Lambda_{n}}|\nabla u|^{2} \geq \frac{\varepsilon}{D n \cosh \varepsilon}
$$

Therefore

$$
\int_{\mathcal{R}}|\nabla u|^{2} \geq \frac{1}{2} \sum_{n \geq n_{0}} \frac{\varepsilon}{D n \cosh \varepsilon}=\infty,
$$

and so $u \notin H D(\mathcal{R})$.

This proves that $\mathcal{R} \in O_{H D}$.

To prove that $\mathcal{R}$ has a LII we need to precise the metric relationship between $G$ and $\mathcal{R}$. Following Kanai's terminology $[\mathbf{K}]$, we say that an application $\varphi$, not necessarily continuous, between two metric spaces

$$
\varphi:\left(M_{1}, d_{1}\right) \longrightarrow\left(M_{2}, d_{2}\right)
$$


is a "rough isometry" if the following two conditions are satisfied:

(i) There are constants $a \geq 1$ and $b \geq 0$ such that

$$
a^{-1} d_{1}(x, y)-b \leq d_{2}(\varphi(x), \varphi(y)) \leq a d_{1}(x, y)+b,
$$

for all $x, y \in M_{1}$.

(ii) For some $\varepsilon>0$, the $\varepsilon$-neighborhood of $\varphi\left(M_{1}\right)$ covers $M_{2}$.

A metric space $M_{1}$ is said to be "roughly isometric" to a metric space $M_{2}$ if there exists a rough isometry from $M_{1}$ into $M_{2}$. Obviously being roughly isometric is an equivalence relation between metric spaces.

It is evident that the graph $G$ and the surface $\mathcal{R}$ are roughly isometric.

If $F$ is a graph, for a subset $P$ of vertices of $F$ we define its "boundary" $\partial P$ by

$$
\partial P \equiv\{v \in V(F): d(v, P)=1\} .
$$

If $|\cdot|$ denotes the cardinal of a subset of vertices, the "linear isoperimetric constant" of $F$ is defined by

$$
h(F)=\sup _{P} \frac{|P|}{|\partial P|},
$$

where $P$ ranges over all the non-empty finite subsets of vertices of $F$.

Combining two lemmas of Kanai [K, p. 401] one obtains that the surface $\mathcal{R}$ and the graph $G$ verify a LII simultaneously. Moreover, the definition of the linear isoperimetric constant in a graph implies that $G$ has a LII if the binary tree $T$ has a LII, because both have the same vertices and $G$ has more edges.

It is not difficult to prove, by induction in the number of vertices of $P$, that

$$
|P| \leq|\partial P|
$$

for all non-empty finite subsets of vertices $P$ of $T$. This complete the proof that $\mathcal{R}$ has a LII.

\section{References}

[AS] Ahlfors, L. V. ANd Sario, L., "Riemann surfaces," Princeton University Press, Princeton, 1960.

[B] Buser, P., A note on the isoperimetric constant, Ann. Sci. Ecole Norm. Sup. (4)15 (1982), 213-230. 
[C] Chavel, I., "Eigenvalues in Riemannian geometry," Academic Press, Orlando, Fla., 1984.

[Ch] Cheeger, J., A lower bound for the smallest eigenvalue of the Laplacian, in "Problems in Analysis," Princeton University Press, Princeton, 1970, pp. 195-199.

[F] FENCHEL, W., "Elementary geometry in hyperbolic space," Walter de Gruyter, Berlin, 1989.

[FR] Fernández, J. L. And Rodríguez, J. M., The exponent of convergence of Riemann surfaces. Bass Riemann surfaces, Ann. Acad. Sci. Fenn. Series A. I. 15 (1990), 165-183.

[K] KANAI, M., Rough isometries and combinatorial approximations of geometries of non-compact Riemannian manifolds, J. Math. Soc. Japan 37 (1985), 391-413.

[R] Randol, B., Cylinders in Riemann surfaces, Comment. Math. Helv. 54 (1979), 1-5.

[SN] SARIO, L. AND NAKAI, M., "Classification theory of Riemann surfaces," Springer-Verlag, Berlin, 1970.

[S] Sullivan, D., Related aspects of positivity in Riemannian geometry, J. Diff. Geom. 25 (1987), 327-351.

[T] TsusI, M., "Potential theory in modern function theory," Maruzen, Tokyo, 1959.

Universidad Carlos III de Madrid

Escuela Politécnica Superior

C/ Butarque $\mathrm{n}^{\circ} 15$

28911 Leganés (Madrid)

SPAIN

Rebut el 10 de Gener de 1994 